\title{
El aprendizaje cooperativo por proyectos: una aportación a la didáctica de la música en la educación universitaria de magisterio en el grado de infantil
}

\section{The cooperative learning projects: a contribution to music teaching in higher education, in the pre-primary education grade}

\author{
Ana María Botella-Nicolás ${ }^{1}$ iD, José Rafael Adell-Valero
}

Tipología: artículo de investigación científica y tecnológica

Para citar este artículo: Botella-Nicolás, A. M. y Adell-Valero, J. R. (2018). El aprendizaje cooperativo por proyectos: una aportación a la didáctica de la música en la educación universitaria de magisterio en el grado de infantil. Praxis, 14(2), 135-154. Doi: http://dx.doi.org/10.21676/10.21676/23897856.2668

Recibido en julio 6 de 2018

Aceptado en septiembre 14 de 2018

Publicado en línea en noviembre 30 de 2018

\section{RESUMEN}

El artículo muestra una investigación sobre la didáctica de la música, realizada de manera experimental con alumnado de enseñanza superior universitaria de Educación Infantil, mediante el aprendizaje con el Método Cooperativo por Proyectos. La eficacia del Método, se ha comprobado mediante dos grupos de alumnos: uno al que se impartieron los contenidos con el citado Método, y otro a los que se les asignaron los mismos contenidos mediante la Clase Magistral. El resultado de la experiencia se mide con pruebas objetivas: un pretest y un postest. También se efectúa una encuesta de percepción con objeto de conocer las apreciaciones subjetivas del alumnado, alrededor de la experiencia vivida. Todas estas pruebas avalan la idoneidad del Método Cooperativo por Proyectos en cuanto a la asimilación de contenidos, objetivos y didáctica, así como su efectividad respecto a la cooperación grupal, activa y sensible y la socialización.

Palabras clave: música; educación infantil; método cooperativo por proyectos; educación superior.

\begin{abstract}
The article shows a research on music teaching, performed experimentally with university students of Pre-primary Education, by learning through the Cooperative Method for Projects. The effectiveness of the method has been checked by two students groups: one who had content delivered with this method, and one to which it was assigned the same content through a Master Class. The result of the experience is measured by objective evidence: a pretest and postest. A perception survey is also carried out in order to make a subjective assessment of students regarding the experience. All these tests support the suitability of the Method for Cooperative Projects concerning the assimilation of content, objectives and teaching, as well as its effectiveness regarding the group's active and sensible cooperation and socializing.
\end{abstract}

Keywords: Music; Kindergarten; Cooperative Learning Method; University.

${ }^{1}$ Pd.D. en Pedagogía. Universitat de Valéncia, Valéncia, España. Correo electrónico: ana.maria.botella@uv.es ORCID: 0000-0001-5324-7152

${ }^{2}$ Ph.D. en Didáctica. Universitat de Valéncia, Valéncia, España. Correo electrónico: radell@florida-uni.es ORCID: 0000-0002-2888-8641 


\section{INTRODUCCIÓN}

a educación musical sigue siendo una preLocupación constante para los docentes que quieren darle la importancia que le corresponde en el currículum, tanto en los niveles elementales de aprendizaje como en la enseñanza universitaria. En la actualidad, la Música está reconsiderando sus objetivos; el más destacado, el que se refiere a su función como elemento integrador de la personalidad, atiende a la formación de los alumnos como seres humanos sensibles, autónomos y creativos que puedan dar respuesta a los interrogantes que plantea la sociedad actual. Esto reafirma la necesidad de una continua renovación a la que debe adaptarse el profesorado de cualquier ámbito, disciplina o nivel.

Es pues, imprescindible, actualizar la metodología de la Música, sobre todo en las facultades universitarias de Magisterio, ya que si en ellas los alumnos se encuentran en una fase de preparación y de estudio que cuando ésta finalice, han de estar en condiciones de proyectar sus conocimientos en las etapas de Infantil y Primaria. La doble vertiente educativa obliga al replanteamiento, no sólo los contenidos que se deben impartir, sino también su aplicación didáctica en las mencionadas etapas. ¿Cómo hacerlo?

No es deseable que la enseñanza se imparta a los alumnos como si ellos fueran niños, con el pretexto de enseñarles a vivenciar los aspectos didácticos más adecuados. Esta forma, ni es razonable, ni se corresponde con la realidad que afecta a la competencia de cada edad. Hay que ejemplificar, pero nunca mimetizar. Por el contrario, a cada nivel universitario hay que asignarle una experiencia específica, de manera que los objetivos, los contenidos y las metodologías sean adecuados a sus edades y a sus necesidades personales y cognitivas.
Escribano (1995) señala que:

Todo ello se expresa en lo que denominamos estrategias de enseñanza, las cuales se refieren a las técnicas de acción que se utilizan en el proceso de enseñar y que van controlando las distintas variables que inciden en un diseño curricular de una disciplina (p.94).

Escribano (1995) especifica que:

Hay tres ejes que son prioritarios en la formación del profesor/a universitario: El estudio, la docencia y la investigación [...] Es decir, profesores que estudian, que enseñan y que investigan; tres dimensiones que habrán de ir conjuntamente en la base inicial de su formación (p.93).

Desde estas premisas se ha trabajado en la investigación de una experiencia activa, cuantitativa y cualitativa, en la que se ha aplicado y contrastado el Método Cooperativo por Proyectos en un contexto real. Para comprobar su eficacia se dividió al alumnado en dos grupos: uno funcionó como control con una metodología de referencia: la Clase Magistral. El otro, considerado como grupo de estudio trabajó mediante el citado Método Cooperativo por Proyectos. Como herramienta de contraste se utilizaron unas pruebas objetivas: Pretest y Postest que permitieron determinar los niveles de contenidos adquiridos con una y otra metodología. Tras un análisis estadístico de los resultados se pudieron extraer conclusiones cuantitativas que se señalarán más adelante. Por otro lado, también se realizó un estudio cualitativo a través de una encuesta de percepción que puso de manifiesto las apreciaciones subjetivas del alumnado en torno a la experiencia vivida. La población a la que se ha sometido la aplicación del método está compuesta por el alumnado de $3^{\circ}$ de Educación Infantil en la asignatura de Procesos musicales del Centro de Florida Universitaria, adscrito a la Universitat de València y a la Universidad 
Politécnica de Valencia con las que mantiene sus títulos homologados.

La asignatura de Música ha figurado en todos los planes de estudio de la Facultad de Magisterio de la Universitat de València (España) desde sus inicios como Escuela Normal hasta la actualidad; aunque al comenzar su andadura en 1857, y durante más de un siglo, su contenido estuvo relacionado con los estudios del Conservatorio, si bien con un rango mucho más elemental. Tuvo que llegar el plan de 1967, para que se cambiaran los contenidos por otros más actualizados, postergándose el solfeo tradicional en favor del desarrollo del sentido del oído, el del instinto rítmico y la sensibilidad, tratando de que se acentuara el placer estético y el deleite musical.

A partir de ese momento, se evolucionó con gran rapidez. En 1970 la Ley General de Educación (LGE), llamada también Ley de Villar Palasí, permitió que las Escuelas de Magisterio se incorporaran a la universidad, hecho que en Valencia se produjo en 1975. En la Ley de Organización General del Sistema Educativo (LOGSE) de 1990, la Música se integra en el Área de Expresión Artística junto con la Plástica y la Dramatización, y en 1991 se crea el título de Maestro-Especialidad de Educación Musical. En la actualidad, la asignatura se contempla en los Grados de Maestro en Educación Infantil y en Educación Primaria, y consta de unos contenidos actualizados (Botella, 2010).

El 1 de enero de 1986 España entró a formar parte de la Comunidad Económica Europea, y sus principales directrices han ido perfilando unos objetivos acordes con dicho marco de referencia internacional. Concretamente, los países miembros de la Organización para la Cooperación y el Desarrollo Económico (OCDE) en 1999, crean el Programme for International Student Assessment (Informe PISA) con el objeto de obtener información sobre los alumnos y determinar si en sus estudios "habían adquirido los conocimientos y el saber hacer esenciales que les permitieran participar de forma plena en la sociedad" (Botella, 2013, p.143). Los resultados obtenidos al respecto por el alumnado español han sido bastante decepcionantes, por cuanto nuestra educación ha estado lastrada en muchos aspectos, por una enseñanza memorística.

Europa siguió elaborando informes de acuerdo con sus nuevas propuestas metodológicas comola Declaración de la Sorbona (1998), la Declaración de Bolonia (1999) y los Comunicados de Praga (2001), y de Berlín (2003). Consensuados los principios fundamentales europeos, que abarcan desde la reordenación de la organización en ciclos de las titulaciones, hasta el modelo educativo que sustenta los métodos de enseñanza; se establecieron una serie de actuaciones que permitieran permeabilizar el espacio común, como por ejemplo: la movilidad académica, la extensión de acuerdo de reconocimiento mutuo, la construcción de redes de cooperación a escala europea, la generalización de la cultura y los proyectos multinacionales. Todas estas actuaciones se concretan en:

- Los Créditos Europeos, "ECTS: European Credit Transfer System como medio apropiado para promover una mayor movilidad entre los estudiantes" (Zaldívar, 2005, p.98).

- La Acreditación de la Calidad, para el reconocimiento mutuo de los títulos.

Los profesionales de la enseñanza española superior, desean redefinir los planteamientos y situar nuestro el contexto metodológico en el marco internacional que les es afín para entrar de lleno en otras formas de aprendizaje que, acordes con nuestro tiempo, propongan al alumnado otras alternativas de conocimiento y de experiencia. 
Para ello hay que tener en cuenta: la cultura, las competencias básicas y los factores que desarrollan la personalidad; como la percepción, la expresión-comunicación y la creatividad.

Durante el último tercio del siglo XIX nacen una serie de metodologías musicales que aportan nuevas orientaciones y experiencias a la enseñanza de la música, como el método: Dalcroze, Ward, Chevais, Kodaly, Willems, Orff o Martenot.

Desde la década de los sesenta, se acentúa la relación entre pedagogos y compositores para tratar de dar nuevos enfoques y sentar las bases de una pedagogía actualizada de la Música, cuyas teorías modernas y acordes con los tiempos, crean un nuevo campo de acción y de visión. Por ejemplo: George Self en Inglaterra, seguido de Brian Dennis y de John Paynter; en Alemania Lili Friedemann que, en 1969, publica su trabajo sobre Improvisación Colectiva como Estudio y Configuración de la Música Nueva, enseñanza que más tarde seguirá Mauricio Kagel. De 1963 a 1974 en EEUU lideran el movimiento Contemporary Music Project (for Creativity in Music Education), Norman dello Joio, compositor y Robert J. Werner, educador. En Suecia Folke Rabe y Jan Bark crean el primer Taller de Sonido que dará lugar en 1975 al Movimiento de Educación Popular. El canadiense Murray Schafer desde 1965 revolucionó la pedagogía musical partiendo del mundo de los sonidos. Junto a él, otros pedagogos franceses como Delalande (1995) o Reibel (2005), investigan otras alternativas sobre el aprendizaje musical propiciando que se haga de forma más activa, cooperativa, autónoma y consciente. “(...) Al mismo tiempo en los países anglosajones, se perfilan propuestas paralelas como las del psicólogo musical James Mursell, que daba entrada a la creatividad y a la integración educativa" (Oriol de Alarcón, 2012, p.19).
La educación contemporánea pretende actualizar la visión de los maestros, evitando que el objetivo sea la formación de grandes virtuosos. El acento se debe establecer en la educación integral del ser humano. El aprendizaje de la música debe centrarse en estimular al alumnado en todos los aspectos: emocional, físico, intelectual, ético y estético, en una proyección educativa que trascienda los propios objetivos de la materia. Estos propósitos, a veces, fueron mal interpretados o seguidos de manera poco efectiva ya que, debido a la novedad de los planteamientos, surgieron muchas soluciones que, por su improvisación y falta de sistematización metodológica, no alcanzaron los resultados deseados. La innovación pedagógica musical que se perseguía se resume en:

- Investigar sobre las características, el efecto y la constitución del sonido, natural o artificial.

- Trabajar desde la creatividad, independientemente respecto a la música o relacionándola con otras formas artísticas, vivenciales, culturales...

- Construir la propia música o, en su defecto, aprovechar las oportunidades que ofrece la introducción de nuevos materiales y procedimientos (recursos musicales) para explorar el mundo sonoro, desde otros parámetros expresivos y experimentales.

- Descubrir y valorar el entorno con sus sonidos, ruidos y silencios para sensibilizar al ser humano y hacerlo consciente del mundo que le rodea, a fin de sacar conclusiones que van más allá de lo musical puesto que afectan a la polución sonora y a su regeneración ambiental.

"Ningún lenguaje puede competir con las capacidades de la música para las simultaneidades polifónicas, para los significados múltiples bajo 
la presión de unas formas intraducibles. La capacidad de suscitar emociones, a la vez específicas y generales, privadas y colectivas, excede en mucho a la que posee el lenguaje" (Steiner, 2012, p.24).

Todo ello se expondrá más adelante en el desarrollo de la experiencia.

\section{METODOLOGÍA}

El hecho de que la experiencia desarrollada esté basada en el trabajo por proyectos, presupone una flexibilidad que en ocasiones parece difícil de controlar. Esta aparente imprevisibilidad no significa que el profesor no se haya planteado con anterioridad un modelo didáctico claro y conciso con el cual se puedan sistematizar los elementos más significativos de la actividad. Así pues, los principios, las orientaciones y las predicciones de carácter hipotético realizadas actúan como un plan estructurado que además de estar acorde con el currículum, permiten el diseño de materiales y la orientación la enseñanza.

\section{Los objetivos}

Han sido muy importantes en este modelo procesual puesto que se entienden como punto de partida reflexivo que aporta grandes ventajas por cuanto son la base de:

- La programación del currículo.

- La comunicación y el trabajo en común (profesores y alumnos).

- La evaluación que afectará a estudiantes, recursos, estrategias y metodología empleados.

- La formación de los alumnos.

También aportan contenidos y argumentos que deben figurar en la base del proyecto a realizar, por lo que serán pertinentes en la elaboración del tema. De cualquier manera, si se pretende conseguir respuestas creativas e innovadoras, será difícil desarrollar objetivos fácilmente identificables.

Según Nisbet y Shucksmith (1987), es indispensable que las estrategias de aprendizaje incluyan también la práctica de procedimientos y de materiales: su clasificación, utilización y construcción. Así mismo, las actitudes de los alumnos han de ser consideradas en relación con su grado de implicación en el grupo, puesto que se impone una visión crítica y reflexiva que oriente sobre sus comportamientos durante la realización de la actividad. Finalmente, las técnicas de trabajo son primordiales para una organización con coherencia y efectividad. En cualquier caso, debe quedar explícito lo que se desea conseguir y el tipo de situaciones formativas que se quieren crear. Por todo lo dicho, los objetivos están directamente relacionados con la evaluación.

Además del conocimiento que adquieran los alumnos, también es fundamental, que manifiesten su capacidad para hacer uso de él, incluso en situaciones nuevas que no se limiten al ámbito escolar. Proyectar las experiencias hacia el entorno y la naturaleza y descubrir similitudes con su experiencia musical sería un gran logro.

Como ya se ha señalado, en esta investigación el principal objetivo ha consistido en contrastar dos métodos de aprendizaje: uno tradicional, apoyado en la Lección Magistral, y otro experimental, aplicando el Método Cooperativo por Proyectos. Con esta premisa se establecen unos planteamientos cuya revisión, confrontación y análisis, en definitiva, constituyan una base empírica que afecte a posteriores actuaciones metodológicas y educativas. Se deberá incidir en tres aspectos importantes: 
- Profundizar en la investigación de nuevas metodologías aplicadas a los programas oficiales de manera que el futuro profesor encuentre los fundamentos básicos que debe impartir al acabar sus estudios de grado.

- Introducir aspectos metodológicos poco explorados que actualicen las estrategias que deben utilizarse para llevar a cabo los contenidos prescritos con la máxima eficacia y diversificación en la Comunitat Valenciana.

- Generar unas dinámicas y uso de herramientas metodológicas en los futuros formadores, que en el momento de su quehacer laboral actúen como una vivencia que se proyecte hacia sus futuros alumnos.

\section{Los contenidos}

Se han contemplado como el capital intelectual, emocional y técnico del cual se van a partir. Por tanto, los contenidos de la asignatura estarán formados por el conjunto de conocimientos, habilidades, lenguajes y valores referidos a la Música. Podemos agrupar los contenidos en dos grandes categorías:

a) Los que pertenecen a la propia Unidad Didáctica.

b) Aquellos que se derivan de las necesidades que se crean cuando el alumno trabaja en grupo.

Ha sido muy efectivo aprovechar su carácter funcional que se proyecta hacia conceptos, destrezas, valores, actitudes y aspectos emocionales. De la misma manera que los objetivos, también se deberán interpretar en forma de competencias.

En la toma de decisiones que se hace antes de cualquier actuación, hay que especificar el tema que se va a tomar como objeto de estudio. Se ha considerado que el ritmo es un pilar básico en las composiciones musicales y, al mismo tiempo, está presente en cualquier manifestación de la naturaleza orgánica, la cual, por estar viva, siempre se encuentra en movimiento. Por tanto, después de reflexionar sobre el contenido del currículo, se ha decidido optar por este tema como objeto de actuación y de investigación. Sus características como ritmo regular y como irregular se han ampliado con el concepto de relatividad (Willems, 1993); es decir, una relatividad entre movimiento y orden que cumple con unos elementos subjetivos como la sensibilidad, la humanidad, la fisiología y la irregularidad, (siendo esta última la que diferencia el ritmo de la rítmica).

Sentir, comprender, vivenciar, imaginar, construir y expresar son algunos de los factores que resultan imprescindibles en una educación integral. Se observa pues, que el ritmo, tanto en la enseñanza universitaria como en Infantil $y$ Primaria es un elemento configurador de situaciones, cuya estructura y profundidad le hacen imprescindible en cualquier proceso de organización constructivo y vital. En este caso se trabajaron con la obra Marcha cumbia de Stephen Tillapaugh, ya que permite el empleo de diferentes instrumentos de percusión de altura determinada e indeterminada y una vivencia rítmica que puede ser de gran interés en cualquier edad.

\section{La evaluación}

Este es también un proceso clave en el sistema educativo. Ha de ser formativa y continua de manera que relacione las opciones tomadas en el resto de elementos del diseño curricular. No se puede prescindir de ella al comienzo, durante, ni al final del proceso educativo por lo que, en este sentido, condiciona la programación, la 
impartición y el resultado final de la actividad. Deberá cumplir con dos funciones básicas como autoevaluación de la propia labor didáctica, y de la validez de las estrategias utilizadas en las que actuará como fuente de retroalimentación. Según Gimeno (1981) como una información que le ayude al alumno a progresar hacia el aprendizaje, ofreciéndole noticias y razones del estado en que se encuentra. De esta manera, él mismo, podrá tomar este dato como guía de autodirección, meta de toda educación.

Sin embargo, no hay que limitarse a estas dos funciones propuestas porque con ser muy importantes no son suficientes. La evaluación debe encontrar nuevas pautas que supongan una tarea investigadora acorde con la actualización del método que se está desarrollando. Además, la evaluación no sólo debe atender a la valoración del progreso de los alumnos, sino que debe afectar a los procesos de la enseñanza-aprendizaje y, particularmente, a los propósitos que los guían y las condiciones en que se desarrollan. Finalmente se deberá tener en cuenta, por un lado, la forma en que se llevará a cabo la evaluación, y por otro, la manera en que esa evaluación se convertirá en calificación.

Aunque parezca que se trabaja con cierta aleatoriedad, fruto de una evaluación no convencional, es necesario que además de el "qué" se evalúa, se preste atención al "cómo" se evalúa puesto que cada alumno puede presentar características diferentes de los demás. En cualquier caso, es importante establecer un compromiso de colaboración mutua entre el docente y el discente; una dimensión diagnóstica, una orientación hacia la mejora, un espacio crítico y creativo para el encuentro, el diálogo, el intercambio integrador y la reflexión compartida. Es por ello que el proceso de evaluación debe ser enriquecido con diversas modalidades como son: la coevaluación, la autoevaluación y la heteroevaluación.
Dentro del proceso de evaluación hay que reconocer que la incorporación de las TIC es un elemento facilitador y de considerable importancia ya que mejora las técnicas y permite, por ejemplo, observaciones que antes no eran posibles. Disponer de una grabación en la cual los mismos alumnos se vean actuando, es una experiencia muy gratificante que les hace ser más conscientes de sus intervenciones. Ello ayuda a objetivar los procesos de autoevaluación y coevaluación. Este recurso, además, hace que el proceso se desarrolle de manera continua y permita al profesor, y a los mismos alumnos, una valoración de sus actuaciones y de sus aportaciones. El acto evaluador se convierte en sí en un proceso múltiple que implica mucho más que medir ya que significa entender, asimilar y avanzar.

La observación sistemática parcial y grupal, permite descubrir algunas capacidades como: organización, reflexión, actuación y crítica de las actuaciones. "Hay mucho que aprender acerca de las aulas y las escuelas mediante la visión de su aspecto real" (Eisner, 1987, p.143).

En el caso de esta experiencia, la evaluación se ha visto totalmente condicionada por la necesidad de objetivar el proceso de investigación que consiste en comparar ambas metodologías. Es por ello que sólo se ha tenido en cuenta el nivel de conocimientos previos (pretest) y el nivel de conocimientos adquiridos después de la intervención (postest). Dado que la enseñanzaaprendizaje es un proceso multifactorial, sólo así se ha conseguido aislar parte de las variables y poder comparar de manera fidedigna ambas metodologías. Con objeto de compensar este déficit importante en el proceso de evaluación se ha incorporado una encuesta de percepción que además de recoger las apreciaciones subjetivas del alumnado sobre la experiencia realizada, enriqueció el proceso evaluador. 


\section{El método cooperativo por proyectos}

No existe la denominación de este método como tal. En realidad, se encuentran separados el Aprendizaje Cooperativo y el de Proyectos, aunque sea posible su refundición. En este caso concreto, ambos métodos se han combinado complementándose ya que juntos, pueden ofrecer un mayor número de posibilidades educativas, al no ser excluyentes.

En principio, el Método Cooperativo es más que un agrupamiento de personas puesto que hay que integrar individuos aislados que comparten espacios físicos y objetivos genéricos. Además, sus componentes deben adoptar roles funcionales, establecer una comunicación fructífera y aunar esfuerzos para obtener resultados óptimos. En España conviene destacar "los trabajos pioneros de Ovejero (1990) o de Echeita y Martín (1990), Echeita (1995), o los más recientes de Díaz Aguado (2003), David Durán (Durán y Vidal, 2004), Pere Pujolàs $(2004 ; 2009)$ o Moruno, Sánchez y Zariquiey (2011)" (Torrego y Negro, 2012, p.26).

En realidad, esta forma de aprendizaje se caracteriza por encontrar la manera de que el rendimiento, tanto individual como grupal, sea totalmente eficaz al proponer y reforzar la cooperación que, como se verá más adelante es un sistema de conseguir resultados educativos que, de otra manera, no es posible obtener. Ovejero (1993) explica que:

La implementación en las escuelas de las técnicas de aprendizaje cooperativo son altamente eficaces para mejorar [...] la motivación intrínseca, la autoestima y el funcionamiento de las capacidades intelectuales, aumentando particularmente las capacidades críticas y la calidad del procesamiento cognitivo de la información (p.387).
En palabas de Pujolàs (2012):

Tratándose de equipos de aprendizaje cooperativo el primer objetivo es obvio: progresar todos en el aprendizaje; saber, al final de cada unidad didáctica y al final de un curso académico determinado, más de lo que sabían al iniciarlo, cada uno según sus capacidades; no se trata de que todos aprendan lo mismo, sino de que cada uno progrese todo lo que pueda en su aprendizaje. Y tratándose de equipos de aprendizaje cooperativo, el segundo objetivo es igualmente claro: ayudarse unos a otros, cooperar, para progresar en el aprendizaje (p.92).

El Método de Proyectos considera una visión de la educación en la cual los alumnos adquieren una mayor responsabilidad en el proceso de su aprendizaje, al aplicar, en proyectos reales, las habilidades y conocimientos adquiridos. Dicha metodología se fundamenta en gran medida en el socio-constructivismo según el cual el aprendizaje se genera a partir de los conocimientos previos de cada uno, por lo que el aprendizaje es significativo en tanto en cuanto se apoya en un sustrato ya existente. Autores como Vygotski (1990) refuerzan la idea asociándola al aprendizaje en grupo, de manera que los alumnos aprenden más rápidamente de forma cooperativa que individualmente.

Este enfoque contempla la cooperación no sólo entre alumnos, sino también entre éstos y sus profesores, lo cual crea una dinámica en la que todos los participantes obtienen beneficios del proceso de aprendizaje. Esto constituye un avance esencial, dado que los participantes tienen más posibilidades de explotar al máximo su autonomía y capacidad de aprender, lo cual fomenta una serie de respuestas psicológicas que favorecen la curiosidad y la creatividad.

Como se ha indicado, hay muchas coincidencias y sinergias importantes entre ambos sistemas 
metodológicos, como por ejemplo, el hecho de que las dos metodologías son abiertas y fomentan en el alumno la curiosidad, la experimentación y el desarrollo propio de estrategias para afrontar las diversas dificultades que aparecen en el proceso de aprendizaje. Además, en ambas, el profesor juega un rol de acompañante o guía en un proceso de metacognición.

\section{Descripción de la experiencia musical en la educación infantil}

Antes de comenzar con la descripción pormenorizada de las siete sesiones que constituyen el eje temporal de la experiencia, convendría apuntar algunos factores destacables en las características del grupo objeto de estudio.

Como es habitual, en Educación Infantil aproximadamente el $98 \%$ del alumnado pertenece al sexo femenino. Ciertamente en los estudios de Magisterio el número de mujeres siempre sobrepasa al de varones, pero en general nunca se da en esta proporción tan exagerada. Dado que las alumnas se encuentran en $3^{\circ}$ curso del Grado de Maestro/a en Educación Infantil, vivieron la experiencia con un gran sentido crítico.

\section{1ªsión}

- Desde el inicio el profesor avanzó cuáles eran sus intenciones, al proponer esta forma de trabajo. Resumiéndolo escuetamente, algunos de los puntos que se comentaron fueron:

- Vamos a trabajar con una Metodología Cooperativa por Proyectos.

- Lo que se consiga, lo que obtengamos durante el proyecto y su realización, se verá reflejado durante el proceso, al margen de que después exista un resultado final. Éste puede ser mejor o peor estética y musicalmente, pero lo relevante será la consecuencia de un trabajo reflexivo en el que intervendréis todas en la medida de vuestras posibilidades. Lo que se pide es que cada una aporte lo que sea capaz de hacer y de comprender, y de ese modo, nos enriqueceremos simultáneamente.

- Vamos a hacer Música con instrumentos no convencionales; construiremos hidrófonos con botellas de vidrio.

- Trabajaremos por grupos cooperativos, pero es evidente que cada cual será responsable de su parte y actuación dentro de los mismos; se espera que cada uno desarrolle sus capacidades, a partir de sus conocimientos previos que en cada caso son diferentes. Por tanto, vamos a trabajar de una forma cooperativa y, también, en cierta medida, autónoma.

- Deberéis adoptar y consensuar acuerdos y decisiones diversas que irán tomando forma a lo largo del proceso.

- El profesor va a actuar como un guía o apoyo cuando aparezcan problemas o se necesite encontrar soluciones puntuales, pero seréis vosotras quienes vais a tratar de resolver las dificultades que se presenten, encontrando soluciones diversas y fomentando el pensamiento divergente.

Además, se añadió que durante la experiencia debían mantener:

- Una actitud positiva frente a los posibles errores que cometieran, ya que de ellos se aprende tanto o más que de los aciertos.

- Apertura a la percepción y autoconfianza.

- Agilidad y persistencia para conseguir los logros propuestos. 
- Concentración y actitud proactiva en todo el proceso, que además favorezca la motivación pues este es un punto fundamental en la Educación Infantil.

También se explicó y justificó brevemente el sistema de evaluación en base a la necesidad de objetivar los resultados obtenidos y poder comparar ambas metodologías.

Pasado el Pretest, se propuso el tema que se iba a trabajar: "El ritmo". El profesor también presentó la partitura: Marcha cumbia, de Stephen Tillapaugh, que debía ser interpretada por ellas. Esto suscitó diversas reacciones en el alumnado, ya que algunas se sentían incapaces de descifrarla. Hubo que tranquilizarlas aclarando que su lectura no iba a ser un problema porque las alumnas que tuvieran conocimientos musicales más avanzados podrían prestarles ayuda.

A continuación, se les pidió que formaran tres grupos para trabajar cooperativamente. Al hacerlo, como es natural, se unieron las que tenían algún tipo de amistad o de afinidad. El profesor tuvo que hacer algún reajuste para compensar las deficiencias que podían producirse y para situar, estratégicamente, entre los grupos, a las alumnas avezadas en la lectura de la partitura.

Así pues quedaron organizadas del siguiente modo:

- Las que iban a tocar los hidrófonos de primera voz.

- Las que iban a componer los hidrófonos de segunda voz.

- Y las que se harían cargo de los tubos y de la pequeña percusión.
Después, se especificó que aunque la actividad estaba organizada y programada para ellas, de acuerdo a su edad, a su situación en la carrera $y$ a sus propios intereses, al final de la experiencia, habría una ejemplificación de cómo se podía trasladar a las edades infantiles en las que ellas deberían actuar en el futuro. Si para ellas iba a ser algo muy enriquecedor, aún lo debía ser más en el contexto infantil donde hay que introducir a los niños en un mundo abierto a muchas posibilidades expresivas en las que deben desarrollar aspectos educativos muy importantes como la atención, la coordinación mental y motriz y la cooperación con las restantes personas.

\section{2a Sesión}

Previamente el profesor había aportado el material con el cual iban a trabajar: las botellas con las que construirían los hidrófonos, los tubos afinados y la pequeña percusión. Hubiera sido deseable que las alumnas hubieran recogido ellas mismas dicho material, pero por cuestiones de tiempo y de planificación no fue posible.

Trabajar con unos materiales reciclados provocó cierta sorpresa ya que parecía imposible que pudieran arrancarse sonidos coordinados con un material tan cotidiano. No obstante, es un aprendizaje social importante por cuanto se debe aprovechar todo lo que habitualmente se desecha como inútil para darle otra finalidad aplicándolo en situaciones como la que nos ocupa.

Las botellas se limpiaron y se procedió a su afinación llenándolas de agua hasta un determinado nivel. El profesor había preparado un hidrófono que sirvió de referencia. Por ello, la afinación de los instrumentos se hizo sin problemas, aunque fue muy laborioso encontrar el sonido adecuado llenándolas o vaciándolas de líquido. Se crearon un total de 8 hidrófonos. En el proceso de afinación se hizo evidente que este ejercicio, que 
parece bastante fácil, necesita de la cualidad auditiva necesaria para determinar la altura de la nota que se busca. Esto es fundamental por cuanto desarrolla su percepción sonora.

Creados los hidrófonos, se probó cada nota en conjunto y por separado, bajo la supervisión del profesor; así como la escala para comprobar su correcta afinación. Hubo que retocar el nivel de agua en muchos casos. Finalmente, la clase acabó con el precintado de botellas para evitar que la evaporación alterara el resultado conseguido.

\section{$3^{\text {a }}$ Sesión}

Se crearon los nuevos grupos llamados "de expertos" en base al diagrama siguiente, tal y como se aprecia en la figura 1:

Figura 1. Desarrollo del intercambio de grupos.

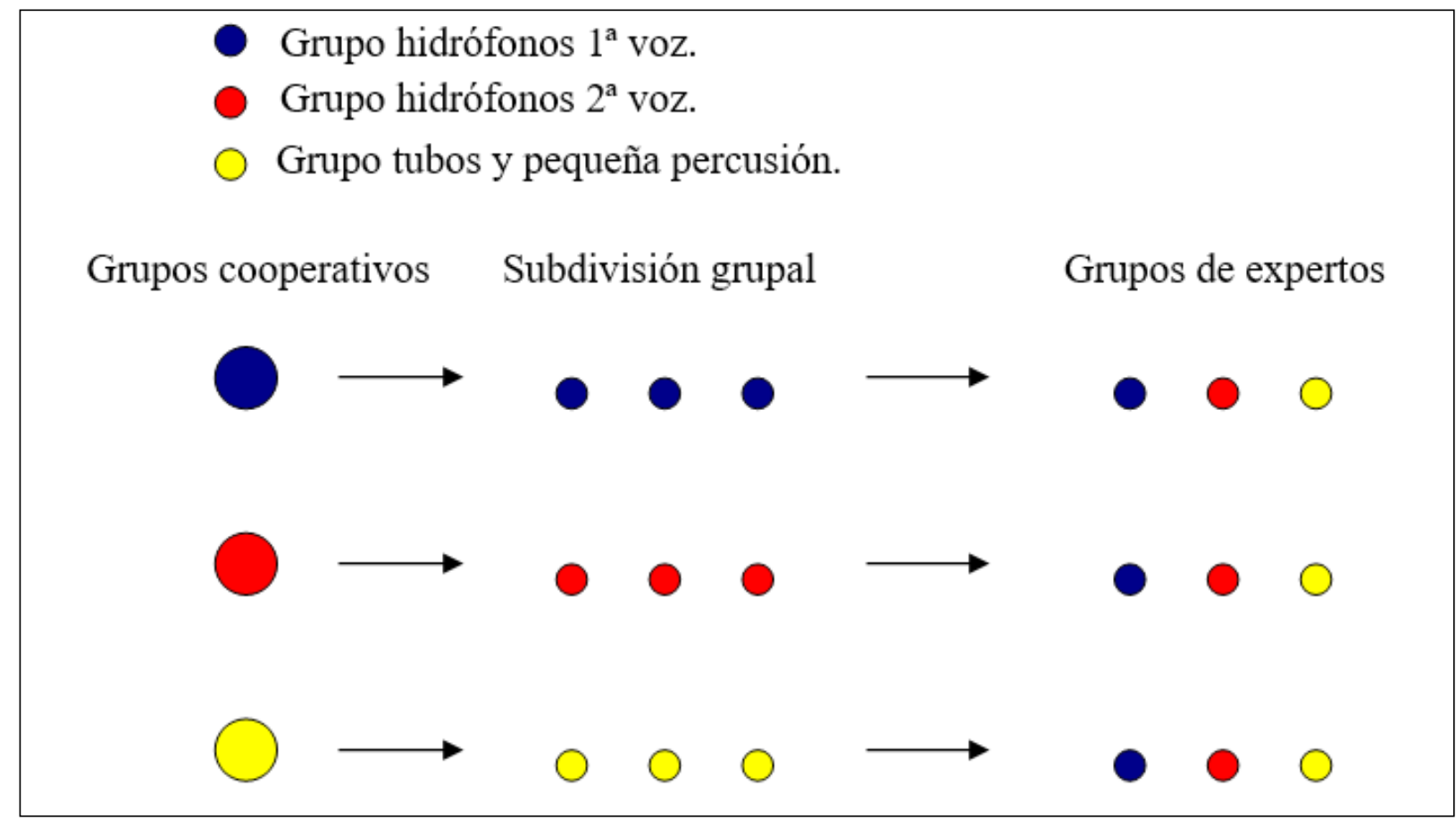

Fuente: elaboración propia.

Los grupos de expertos están formados por alumnas de cada uno de los grupos iniciales, de manera que cada una puede aportar su visión parcial de lo trabajado hasta el momento, facilitando así la comprensión global de la obra. Una alumna preguntó por qué había elegido el profesor esta obra; una música de carácter popular escrita por un compositor extranjero. Explicó entonces sus características relacionadas con el ritmo, la melodía, su composición relativamente fácil de interpretar; sobre todo insistió en la conveniencia de ampliar el campo sonoro hacia obras menos conocidas que, de alguna forma, ampliaban su visión musical de entornos cercanos.

Por supuesto hay que conservar nuestras raíces, desarrollarlas y recurrir a ellas para mantener nuestra propia identidad, pero corremos el 
riesgo de sufrir un empobrecimiento si actuamos con estas limitaciones tan constrictivas. Un ciudadano del siglo XXI debe mantenerse en su cultura, pero también abrirse a otras que, más pronto o más tarde, enriquecerán su mundo artístico con aportaciones que serán estímulos importantes en el desarrollo de una personalidad integrada en el mundo cultural. El arte, cuando es auténtico, es universal y expone una forma de libertad sin condiciones que puede actuar muy positivamente en nuestro desarro- llo personal. Abrir y no cerrar, es la clave para una realización consecuente.

\section{$4^{a}$ Sesión}

Llegados a este punto, además de la melodía, las alumnas pudieron apreciar los aspectos rítmicos y estructurales de la obra. Para ello, el profesor dibujó un esquema de cómo se integraban las voces en el transcurso de la composición, para que ésta tuviese sentido (figura 2).

Figura 2. Integración de las voces en la obra.

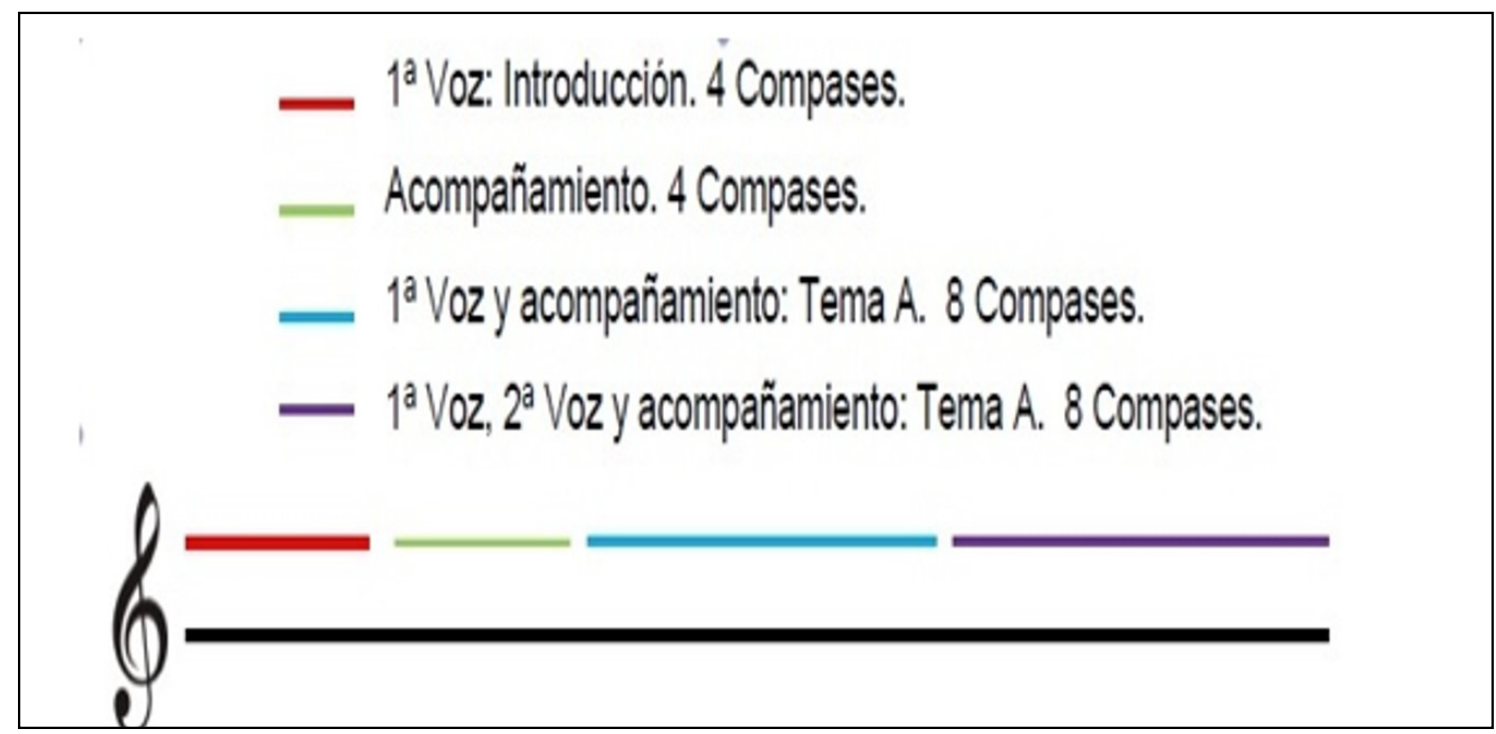

Fuente: elaboración propia.

De este modo los momentos de silencio (compases de espera) cobran sentido y se entiende el juego de texturas que ha diseñado el compositor. Actuar ordenadamente para conseguir efectos y subordinar sus actuaciones respetando el trabajo de los demás fue algo que se iba consolidando y consiguiendo lentamente. Ahora era posible ir reconociendo el resultado sonoro con la partitura. Además, los signos escritos tenían un significado concreto y se aprovechó para identificarlos reconociendo los que son estáticos con los que proporcionan cierto dinamismo a la estructura. A medida que la experiencia se iba consolidando los grupos empezaron a intercambiar ideas y a organizarse para que todos cumplieran su parte en el rol de los demás.

\section{$5^{\text {a }}$ Sesión}

Siempre hay personas más creativas que otras y su talante innovador les lleva a intentar introducir algún cambio significativo en la interpretación de la partitura. El profesor estaba atento a estas intervenciones y procuraba, en lo posible, darles cabida en el desarrollo de la actividad. A tal efecto, sometía al criterio de los demás las 
variaciones (eso sí, mínimas) que las alumnas más creativas pretendían introducir. Entonces detenía el proceso y sometía a consenso, aquellas pequeñas intervenciones. Todo es importante y no hay que desestimar esos aspectos puntuales que se producen.

El análisis es fundamental por cuanto les fuerza a concentrarse y a elegir lo que resulta más conveniente. En ninguno de los casos se eligió la propuesta creativa, aunque el profesor insistió en que había sido un esfuerzo muy interesante y digno de encomio. El consenso también es muy importante porque al mismo tiempo que salvaguarda las ideas de todas las personas y se les da la relevancia que merecen, se hace participar a todo el grupo en una decisión que obliga a analizar e implicarse en los resultados obtenidos. Además de las diversas interrupciones para resolver dudas, el profesor iba parando en determinados momentos para llamar la atención sobre aspectos rítmicos que se debían trabajar en el tema.

\section{Sesión}

Se procedió a hacer un ensayo general. Ya habían superado varias fases; por ejemplo la coordinación motriz, su situación entre los restantes miembros del grupo y, en definitiva, un interés en conseguir que, aquello que al principio les había parecido extraño o poco habitual, se fuera consolidando.

Es notorio que, además de la medida, es necesaria una sensibilización de lo que está escrito en la partitura, una comprensión de sus elementos lingüísticos y una identificación con el espíritu del lugar de procedencia. Cuantas más referencias se tengan de una obra, mayor es la comprensión de la misma. Curiosamente, cuando los alumnos se ven involucrados en la interpretación, se sienten incluidos en el proceso educativo de otra forma. Además, el refuerzo personal de los compañeros, el ir descubriendo que unos benefician a otros, que indagan, investigan y someten a la opinión los demás aquello que para ellos es relevante, actúa como un importante estímulo.

Surgió la necesidad de clarificar qué es sonido y que es ruido, y qué tipos de sonidos deberían estar inmersos en una obra y cuáles no, por considerarlos ruido. Ante esta polémica el profesor explicó en primer lugar cual es la acepción teórica del concepto de sonido y de ruido, haciendo referencia a la regularidad o no en el número de vibraciones por segundo del cuerpo sonoro. Dicho esto, también aprovechó para explicar que incluso un ruido desagradable puede formar parte de una obra si ésta pretende conseguir un determinado efecto, cosa muy habitual en la música contemporánea. Precisamente en las composiciones musicales más modernas se presta atención a estos efectos sonoros que, cuando se está viviendo en la cotidianeidad, resultan molestos y desagradables.

Se volvió a retomar el trabajo y se hizo una grabación parcial de lo que habían conseguido. Debido a que no se disponía de más tiempo sólo pudo construirse una parte reducida de la partitura y todas lamentaron no poder acabar de interpretar toda la pieza; ahora, conociendo el mecanismo y los sistemas de actuación, hubiera sido mucho más rápido finalizar la obra.

\section{$7^{\text {a }}$ Sesión}

El último día, las alumnas se encontraban mucho más relajadas y sus comentarios demostraban que la experiencia había acabado satisfactoriamente. Para comprobarlo se realizó una grabación de todo lo que habían estudiado. Fue una auténtica sorpresa escucharlo.

Hay que tener en cuenta que, para los músicos profesionales, esta experiencia se hace tan 
cotidiana que no necesitan reflexionar sobre ella. Cuentan los compases para intervenir, respetan los silencios, atienden a los movimientos que realiza el director... En resumen, el formar parte de una orquesta, conjunto, es habitual, de sentido ritual y concreta. En este caso, las alumnas aprendieron a tocar juntas, a respetarse, a escucharse, a descubrir el valor de las notas, de los compases, de los signos de puntuación, del ritmo, de la expresividad, del consenso necesario para que la obra sonara bien.

El profesor les propuso acabar por su cuenta la interpretación de toda la obra para que después, al final del curso, hicieran un pequeño concierto en el que, por supuesto, habría que aumentar la emoción expresiva recurriendo al color, a la forma y a la presentación sobre el escenario haciendo incluso una pequeña coreografía. De esta manera, se intensificaría la interrelación con otras áreas como la de plástica, la de expresión corporal, e incluso la de una incipiente representación teatral, que sumarían valores a su experiencia. La clase finalizó con el pase del Postest y de la encuesta de percepción.

\section{Comparación entre las metodologías}

La Lección Magistral responde a:

1- Exposición.

2- Memorización de lo expuesto.

3- Verbalización de lo memorizado (prueba oral o escrita).

\section{4- Feed back sobre el resultado.}

La Lección Magistral es útil cuando se trata de analizar el contenido general del curso, o para explicar los métodos de trabajo de un determinado aspecto del programa, pero resulta incompleta cuando se pretende basar todo el contenido de la programación en ella. La clase magistral es siempre individual y homogénea. Atiende principalmente a la generalización, lo común y lo predecible. En este caso, las pruebas idóneas para la calificación del resultado pueden ser los tests cuya objetividad está íntimamente relacionada con los contenidos. Sistema incompleto cuando se pretenden valorar además actitudes, valores y aprendizajes significativos.

\section{RESULTADOS}

\section{Resultados de las pruebas objetivas: pretest y postest}

La población objeto de estudio fue de 54 alumnas de las cuales 33 realizaron la Metodología Clásica y 21 la de Proyectos. En la siguiente tabla 1 se presentan los resultados de las puntuaciones medias obtenidas por las alumnas antes y después de la intervención:

Tabla 1. Comparación de resultados obtenidos en ambas metodologías.

\begin{tabular}{|l|c|c|c|c|}
\hline Metodología & \multicolumn{2}{|c|}{ Clase Magistral } & \multicolumn{2}{c|}{ Por Proyectos } \\
\hline Test & Pretest & Postest & Pretest & Postest \\
\hline Media & 6,27 & 7,91 & 6,58 & 8,01 \\
\hline
\end{tabular}

Fuente: elaboración propia.

Como se puede observar en la tabla 1 las puntuaciones obtenidas en ambas metodologías para el Pretest y el Postest apenas difieren.

\section{Resultados de la encuesta de percepción}

La encuesta de percepción consta de 10 preguntas (tabla 2). Las nueve primeras cuestiones son cuantitativas y están distribuidas en cuatro bloques. La décima pregunta es cualitativa: 
Tabla 2. Análisis de las preguntas de la encuesta de percepción.

\begin{tabular}{|c|c|c|c|}
\hline & Bloque & Pregunta & Propósito \\
\hline \multirow{9}{*}{ 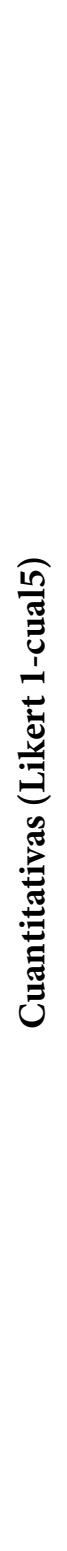 } & \multirow{3}{*}{ 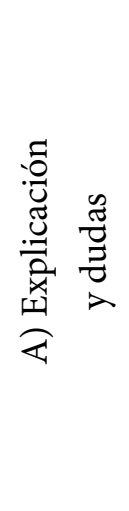 } & $\begin{array}{l}\text { 1. Se ha explicado el trabajo y lo que } \\
\text { se pretendía conseguir antes de iniciar } \\
\text { las actividades. }\end{array}$ & $\begin{array}{l}\text { Comprobar si los alumnos conocen las } \\
\text { tareas a realizar y los objetivos que se } \\
\text { persiguen en las mismas. }\end{array}$ \\
\hline & & 2. Se ha explicado el tema con claridad. & $\begin{array}{l}\text { Verificar si los contenidos se han } \\
\text { impartido en forma y nivel acordes al } \\
\text { alumnado. }\end{array}$ \\
\hline & & $\begin{array}{l}\text { 3. El profesor ha ayudado a resolver los } \\
\text { problemas que se iban planteando. }\end{array}$ & $\begin{array}{l}\text { Cotejar si el profesor domina la mate- } \\
\text { ria y es capaz de resolver las dudas que } \\
\text { se plantean. }\end{array}$ \\
\hline & \multirow{3}{*}{$\begin{array}{l}\frac{\pi}{0} \\
00 \\
0 \\
0 \\
0 \\
0 \\
0 \\
\sum_{0}^{0} \\
\frac{0}{0}\end{array}$} & $\begin{array}{l}\text { 4. Me ha gustado la manera de dar el } \\
\text { tema. }\end{array}$ & $\begin{array}{l}\text { Confirmar el grado de satisfacción } \\
\text { referido a la metodología empleada. }\end{array}$ \\
\hline & & 5. He aprendido a trabajar en grupo. & $\begin{array}{l}\text { Revisar el grado de cooperación e inte- } \\
\text { racción entre alumnos en la metodolo- } \\
\text { gía utilizada. }\end{array}$ \\
\hline & & 6. He disfrutado aprendiendo. & $\begin{array}{l}\text { Comprobar el nivel de satisfacción } \\
\text { conseguido en el proceso de enseñanza } \\
\text { aprendizaje. }\end{array}$ \\
\hline & \multirow{3}{*}{$\begin{array}{l}\frac{\tilde{\Xi}}{0} \\
\frac{0}{0} \\
\text { ล }\end{array}$} & 7. La evaluación ha sido justa. & $\begin{array}{l}\text { Examinar si se percibe el test como una } \\
\text { prueba objetiva de evaluación. }\end{array}$ \\
\hline & & $\begin{array}{l}\text { 8. La evaluación responde al trabajo } \\
\text { que se ha desarrollado en clase. }\end{array}$ & $\begin{array}{l}\text { Observar si el instrumento de evalua- } \\
\text { ción (test) está acorde con la metodo- } \\
\text { logía desarrollada en el aula. }\end{array}$ \\
\hline & & $\begin{array}{l}\text { 9. Recomendarías esta clase a un } \\
\text { compañero/a. }\end{array}$ & Evaluar el nivel de satisfacción general. \\
\hline 苛 & 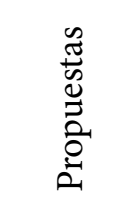 & $\begin{array}{l}\text { 10. Describe tu opinión sobre la } \\
\text { experiencia vivida. ¿Cambiarías alguna } \\
\text { cosa? }\end{array}$ & $\begin{array}{l}\text { Aportaciones destacables y propuestas } \\
\text { de mejora. }\end{array}$ \\
\hline
\end{tabular}

Fuente: elaboración propia. 
Los resultados obtenidos en la encuesta de percepción son los que se muestran en la tabla 3 siguiente:

Tabla 3. Resultados encuesta de percepción parte cuantitativa.

\begin{tabular}{|c|c|c|}
\hline INFANTIL & \multicolumn{2}{|c|}{$\begin{array}{c}\text { Encuesta de percepción } \\
\text { parte cuantitativa }\end{array}$} \\
\hline Preguntas & $\begin{array}{c}\text { Metodología } \\
\text { Tradicional }\end{array}$ & $\begin{array}{c}\text { Metodología por } \\
\text { Proyectos }\end{array}$ \\
\hline $1^{\mathrm{a}}$ & 3,21 & 4,69 \\
\hline $2^{\mathrm{a}}$ & 4,21 & 4,44 \\
\hline $3^{\mathrm{a}}$ & 3,76 & 4,75 \\
\hline $4^{\mathrm{a}}$ & 1,94 & 4,81 \\
\hline $5^{\mathrm{a}}$ & 1,36 & 4,44 \\
\hline $6^{\mathrm{a}}$ & 1,76 & 4,75 \\
\hline $7^{\mathrm{a}}$ & 3,61 & 2,42 \\
\hline $8^{\mathrm{a}}$ & 1,94 & 1,26 \\
\hline $9^{\mathrm{a}}$ & 1,64 & 4,75 \\
\hline MEDIA $^{2}$ & 2,60 & 4,03 \\
\hline
\end{tabular}

Fuente: elaboración propia.

Como se puede observar en la tabla 3, todas las puntuaciones de la Metodología por Proyectos están por encima de las de la Clásica. Sólo hay dos excepciones y se dan en las preguntas relacionadas con la evaluación.

\section{DISCUSIÓN}

Tal y como se ha podido observar en el apartado de resultados, las puntuaciones obtenidas en ambas metodologías para el Pretest y el Postest apenas difieren.

Se llega a la conclusión de que, tal como ha puesto de manifiesto la prueba estadística $t$-student para muestras independientes, los niveles de conocimientos iniciales (Pretest) eran similares; así como los obtenidos después de la intervención (Postest).
Previa a la aplicación del test t-student se pasaron las pertinentes pruebas K-S que hicieron posible la aplicación de dicho test para muestras independientes con la siguiente hipótesis nula $\mathrm{H}_{0}$ : no existen diferencias significativas entre los resultados del Postest (o el Pretest) en ambos grupos.

Los resultados del test estadístico t-student para muestras independientes para los Pretests en Infantil fueron:

$t(52)=-1,333 ; p>0,05$

Dado que la significación es mayor que 0,05

$\mathrm{p}=0,188$

No se pudo rechazar $\mathrm{H}_{0}$ y por tanto se concluyó que no existían diferencias significativas en el Pretest entre ambos grupos. Una vez más se puede considerar que los grupos tienen un nivel similar de conocimientos previos.

Los resultados del test estadístico t-student para muestras independientes para los Postests de Infantil son:

$t(51,928)=-0,450 ; p>0,05$

Dado que la significación es mayor que 0,05

$\mathrm{p}=0,654$

No se puede rechazar $\mathrm{H}_{0}$ por lo que se concluye que no existen diferencias significativas en el Postest entre ambos grupos, por tanto se pueden considerar que se han alcanzado niveles de conocimientos similares en ambas metodologías.

Por tanto, se ha conseguido que las alumnas adquieran prácticamente el mismo nivel de conocimientos usando metodologías diferentes. Tal y como puede observarse en la figura 3: 
Figura 3. Comparación de resultados de la Metodología Clase Magistral con la de Aprendizaje Cooperativo por Proyectos.

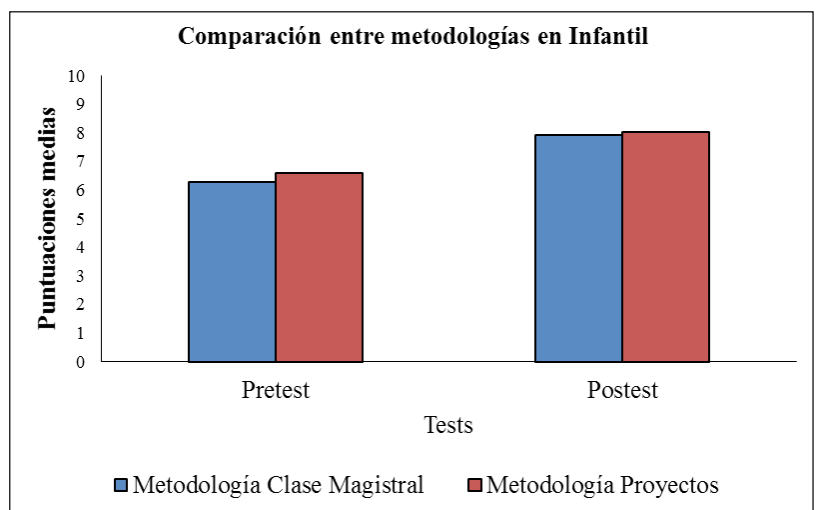

Fuente: elaboración propia.

La comparación entre los resultados del Pretest y el Postest demuestran que en ambos casos las alumnas han mejorado de forma similar tras la intervención. La diferencia entre el Pretest y el Postest para la Metodología Tradicional es de 1,64 puntos, mientras que en el caso del aprendizaje cooperativo por proyectos es de 1,43 puntos. No existen por tanto diferencias significativas entre los niveles de conocimientos obtenidos con una u otra metodología tal y como se ha demostrado con el test ANCOVA.

Respecto a la encuesta de percepción como se puede observar en la tabla 3, exceptuando las preguntas relacionadas con la evaluación, todas las puntuaciones de la Metodología por Proyectos están por encima de las de la Clásica. Con ello las alumnas expresan que la Metodología de Proyectos supera en todos los aspectos a la Clase Magistral, pero demuestran su disconformidad respecto al sistema de evaluación utilizado. Como se ha comentado anteriormente esto fue necesario hacerlo así para poder efectuar una comparación objetiva entre ambas metodologías.
Respecto a la pregunta número 10: "describe tu opinión sobre la experiencia vivida. ¿Cambiarías alguna cosa?"

Los resultados en el caso de la Metodología Clásica apuntan la necesidad de un cambio metodológico. Prevalece la idea de que la clase debería darse de modo más participativo y dinámico. Afirman que sus futuros alumnos necesitarán más elementos motivadores. También señalan que si se dieran las clases de manera más experimental los aprendizajes serían más duraderos, tal y como se aprecia en la figura 4.

Figura 4. Estudio porcentual de temáticas en la pregunta 10 de la Metodología Clásica.

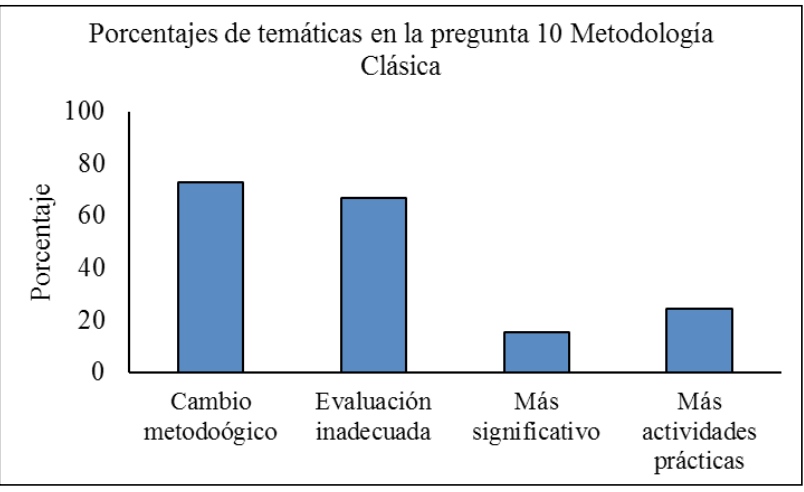

Fuente: elaboración propia.

\section{Proyectos}

El $100 \%$ de las encuestas están a favor de la Metodología por Proyectos. Un 75\% que comenta el sistema de evaluación como muy inadecuado o muy malo para evaluar el trabajo desarrollado en clase. Se debe buscar un sistema de evaluación que tenga en cuenta el proceso y no solo el resultado final, en cuanto a nivel de conocimientos adquiridos, que permita la autoevaluación y la coevaluación. Al igual que en el caso anterior (figura 5), los comentarios son extensos y tratan diversos temas por lo que resulta más apropiado un diagrama de barras para su visualización: 
Figura 5. Estudio porcentual de temáticas en la pregunta 10 de la Metodología por Proyectos.

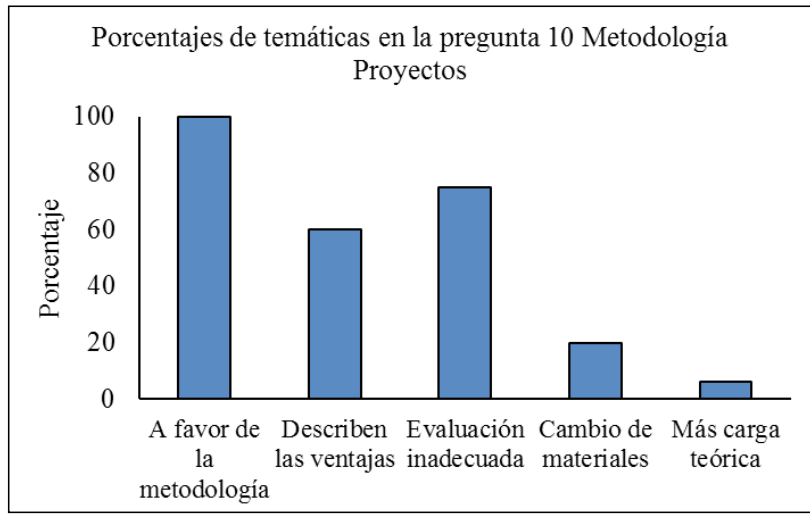

Fuente: elaboración propia.

Como en el caso anterior, todos los comentarios van en la misma línea y apuntan hacia la necesidad de unas metodologías participativas y que atiendan a las características específicas del alumnado a las que van dirigidas. Han de ser flexibles y aplicables a la diversidad del aula.

\section{CONCLUSIONES}

Investigar sobre dos sistemas de aprendizaje distintos no tendría nada de especial si, simultáneamente, no se hubiera dedicado un espacio importante al estudio de la transformación del concepto de la enseñanza musical que se ha producido desde mediados del siglo XX. Como ya se ha apuntado, aunque se disponga de un buen método para impartir la enseñanza, es necesario que éste se adecue a un concepto, a un planteamiento teórico que va a estar en la base del proceso de metacognición. A tal efecto, se adjunta un cuadro (tabla 4) en el que se aprecia la comparativa entre ambas metodologías:

Tabla 4. Comparativa entre la Lección Magistral y por Proyectos.

\begin{tabular}{|l|l|l|}
\hline & \multicolumn{1}{|c|}{ Lección magistral } & \multicolumn{1}{c|}{$\begin{array}{c}\text { Aprendizaje } \\
\text { cooperativo por proyectos }\end{array}$} \\
\hline Rol del profesor. & Fuente de conocimiento. & Guía en el proceso de Metacognición. \\
\hline $\begin{array}{l}\text { Adquisición de } \\
\text { Conocimientos. }\end{array}$ & $\begin{array}{l}\text { A través de una explicación y } \\
\text { posterior memorización. }\end{array}$ & $\begin{array}{l}\text { A través de una experiencia, se } \\
\text { construye conocimiento. }\end{array}$ \\
\hline $\begin{array}{l}\text { Tiempo de realización y } \\
\text { preparación. }\end{array}$ & $\begin{array}{l}\text { Más corto, tanto en la } \\
\text { realización como en la } \\
\text { preparación. }\end{array}$ & $\begin{array}{l}\text { Más dilatado tanto en la realización } \\
\text { como en la preparación. }\end{array}$ \\
\hline $\begin{array}{l}\text { Permanencia de } \\
\text { conocimientos adquiridos. }\end{array}$ & $\begin{array}{l}\text { A menudo a corto plazo y sin } \\
\text { proyección. }\end{array}$ & $\begin{array}{l}\text { A largo plazo. Posibilidad de } \\
\text { transferencia a situaciones diferentes. }\end{array}$ \\
\hline Atención a la diversidad. & Limitada. & $\begin{array}{l}\text { Amplia. El proyecto permite la } \\
\text { inclusión de todo tipo de alumnado. }\end{array}$ \\
\hline $\begin{array}{l}\text { Adquisición de competencias } \\
\text { Básicas. }\end{array}$ & Limitada y poco significativa. & Amplia y socializadora. \\
\hline Percepción de los alumnos. & $\begin{array}{l}\text { Positiva. Pero poco vivencial. } \\
\text { Son público. }\end{array}$ & $\begin{array}{l}\text { Muy positiva. Vivencial. } \\
\text { Son protagonistas. }\end{array}$ \\
\hline
\end{tabular}

Fuente: elaboración propia. 
Hay que remarcar que los estudios de Magisterio no pretenden crear profesionales en el campo de la música, sino a docentes que deberán iniciar a sus propios alumnos en la sensibilización del lenguaje sonoro. Más que una acumulación de conocimientos puntuales, el objetivo del profesor debería ser educar en la adquisición de una forma de expresión y de experiencia personal con la que se podrán enriquecer y desarrollar la percepción, la capacidad de comunicación y la sensibilidad, ampliando los conocimientos respecto al medio y a las distintas áreas que componen el currículo educativo de manera transversal. Así pues, la base de la proyección didáctica es fundamental. Además, el aprendizaje por descubrimiento necesita una gran dosis de imaginación, del sentido de la aventura y del riesgo e incluso el aprovechamiento del azar para estimular opciones que, de otra manera, quedarían relegadas e incompletas. Los aspectos lúdicos y significativos hacen posible despertar en el alumnado la afición, el interés y el aprecio por una asignatura que sobrepasa los simples niveles informativos.

Por tanto, en este trabajo se trata un estudio de caso con una muestra limitada de alumnos, y no pretende que sus resultados sean extrapolables. A pesar de ello, se demuestra la necesidad de seguir buscando metodologías alternativas a la clase magistral para mejorar la didáctica de la música.

Hacer música para aprender prácticamente su constitución y sus mecanismos teóricos, programar actividades que induzcan a actuar, a sentir y a conocer aquello que como lenguaje resulta bastante duro de asumir cuando se siguen métodos tradicionales, es un logro muy importante. Junto a esto, vivir intensamente la experiencia de trabajar en grupo, de manera cooperativa, en la asunción de un proyecto común, es otro logro no menos importante. A todo ello podría añadirse, que para alcanzar resultados óptimos hay que creer en lo que se hace. Y en este caso, se ha seguido, con absoluta convicción y como principio, el espíritu de las teorías de los mencionados autores por considerar que son fundamentales para establecer nuevos replanteamientos metodológicos a fin de investigar y elegir un compromiso real desde la experimentación llevada a cabo desde estos parámetros. Sería deseable que esta experiencia contribuyera a la clarificación de conceptos y animara a proseguir otras investigaciones que, sin duda, serán relevantes y necesarias.

\section{REFERENCIAS BIBLIOGRÁFICAS}

Botella, A. M. (2010). La enseñanza de la música en la Escuela de Magisterio de Oviedo. Magister, 23, 79-90.

Botella, A. M. (2013). La asignatura de música en la Universidad de Valencia: de la Escuela Universitaria a la Facultad Universitaria a la Facultad de Magisterio. Revista Docencia $e$ Investigación, 22, 139-157.

Delalande, F. (1995). La música es un juego de niños. Buenos Aires: Ricordi Americana.

Escribano, A. (1995). Aprendizaje cooperativo y autónomo en la enseñanza universitaria. Enseñanza, 13, 89-102. Recuperado de http:// gredos.usal.es/jspui/bitstream/10366/69492/1/ Aprendizaje_cooperativo_y_autonomo_en_la.pdf

Eisner, W. (1987). Procesos cognitivos y curriculum. Una base para decidir lo que hay que enseñar. Barcelona: Martínez Roca.

Gimeno, J. (1981). Teoría de la enseñanza y desarrollo del currículo. Madrid: Anaya.

Nisbet, J. y Shucksmith, J. (1987). Estrategias de aprendizaje. Madrid: Santillana.

Oriol de Alarcón, N. (2012). Contribución de la enseñanza musical, en los estudios de magisterio en España, a la conservación del arte y la cultura popular. DEDiCA Revista de educação e humanidades, 3, 13-42. 
Ovejero, A. (1993). El aprendizaje cooperativo: Una eficaz aportación de la psicología social a la escuela del siglo XXI. Psicothema, 5, 373-391. Recuperado de http://www.psicothema.com/pdf/1149.pdf

Pujolàs, P. (2012). La implantación del aprendizaje cooperativo en las aulas. En J. Torrego y A. Negro. (Ed.), Aprendizaje cooperativo en las aulas (pp. 77 104). Madrid: Alianza editorial.

Paynter, J. (1999). Sonido y estructura. Madrid: Akal.

Reibel, E. (2005). Lécriture de la critique musicale au temps de Berlioz. Paris: Honoré Champion.

Schafer, M. (2008). El rinoceronte en el aula. Buenos Aires: Melos.

Steiner, G. (2012). La poesía del pensamiento. Madrid: Ediciones Siruela.
Torrego Seijo, J. C. y Negro Moncayo, A. (Comp.) (2012). Aprendizaje cooperativo en las aulas. Fundamentos y recursos para su implantación. Madrid: Alianza editorial.

Vygotski, L. S. (1990). La imaginación y el arte en la infancia. Barcelona: Akal.

Willems, E. (1993). El ritmo musical. Buenos Aires: Eudeba.

Zaldívar, A. (2005). Las enseñanzas musicales y el nuevo Espacio Europeo de Educación Superior: El reto de un marco organizativo adecuado y la necesidad de la investigación creativa y "performativa". Revista Interuniversitaria de Formación del Profesorado, 19(1), 95-122. Recuperado de http://www.redalyc.org/ pdf/274/27419107.pdf 